\title{
Header Bidding as Smart Service for Selling Ads in the Digital Era
}

\author{
Claudia Loebbecke ${ }^{1 \star}$, Stefan Cremer $^{1}$, Maximilian Richter ${ }^{1}$
}

${ }^{1}$ Dept. of Media and Technology Management, University of Cologne, Cologne, GERMANY

*Corresponding Author: claudia.loebbecke@uni-koeln.de

Citation: Loebbecke, C., Cremer, S. and Richter, M. (2020). Header Bidding as Smart Service for Selling Ads in the Digital Era. Journal of Information Systems Engineering and Management, 5(4), em0123. https://doi.org/10.29333/jisem/8483

\section{ARTICLE INFO}

Published: 29 Aug. 2020

\begin{abstract}
Header bidding refers to an innovative, smart service for online advertising. It facilitates concurrent, automatic real-time bidding for ad spaces on online publishers' websites and thereby supports online publishers' in their efforts to tackle Google's dominance in the online advertising market. Interdisciplinary service science research on the opportunities and challenges of smart header bidding services is still scarce. To this end, we analyze a comprehensive dataset, covering header bidding solutions and connected supply-side platforms, for the top 20 German website publishers. We carve out requirements for establishing header bidding as a successful smart service in the digital arena and offer some guidance on how to design and take advantage of header bidding services for monetizing publishers' online content. We further suggest that publishers must monitor rising header bidding substitutes, such as Google's exchange bidding, to secure sustainable value creation from header bidding as technology-based service transformation in the digital world.
\end{abstract}

Keywords: smart services, online advertising, header bidding, content monetization

\section{INTRODUCTION}

Publishers were among the first to benefit from digitization. On the Internet, they can distribute their content such as articles, songs, photos, or videos faster and to a broader audience than via traditional media. Online publishers' two main - partially intertwined - business models are (1) generating direct revenue from selling online content 'per unit' or via subscriptions to the users and (2) generating indirect revenue from display advertising on their sites. However, radically decreasing transaction costs pose a significant challenge to monetizing online content (Shirky, 2008) and erode the online publishers' business models (Cook and Attari, 2012).

In this paper, we focus on publishers selling slots for online display advertising. Here, after digitization has transformed the service offerings (Maglio and Lim, 2016), Google has for years exercised control over the digital advertising industry - latest since its acquisition of what today is 'DoubleClick for Publishers' in 2008. Automated and smart technologies are dominating the business of selling ad services - even if the established basic business model does not change: after selling a slot for online display advertising, an ad is shown on a publisher's website.

On the supply side, a publisher decides on the placement, format, and size of a banner and chooses how to market the available ad impressions in a way that maximizes revenue. On the demand side, an advertiser (brand or agency) buys a certain number and quality of impressions depending on budget and advertising goals. Traditionally, publishers and advertisers contracted directly (Balseiro and Candogan, 2017); in the era of smart services, they match supply and demand - automated and real-time - via an ad exchange (Gonzalvez and Mochon, 2016). Among ad exchanges, publishers can choose between waterfall auctions and header bidding auctions.

The recent rise of header bidding, based on increasingly autonomous technologies, comes across as a publishers' initiative to adjust their online business model in a way that circumvents Google's dominance. In February 2018, about 6.5\% of the top 1,000 websites in the US adopted header bidding - 15\% more than in September 2017 (Serverbid, 2018). In early 2017, German publisher Axel Springer switched its Google-sponsored waterfall-based ad sales technology to a header bidding solution provided by AppNexus. In 2018, they reported a $10 \%$ increase of ad revenue, with the Cost-Per-Mille (CPM) rising by $28 \%$ compared to the previous year (Davies, 2017).

Header bidding requires publishers to adjust their advertising strategy. However, research so far has barely investigated the implications of header bidding for online advertising business models. Therefore, this paper builds on the following research question: What are the challenges of header bidding for online content publishers regarding their advertising-based business models? 
To answer this research question, we analyze the requirements for successful header bidding and summarize the opportunities and challenges from implementing header bidding as a publisher's dominant business model for display advertising.

To this end, we provide an overview of header bidding as a smart service increasingly gaining ground in the advertising market. We lay out the foundations for header bidding research, and provide insights on the header bidding process, wrappers, and platforms. We then analyze the top 20 German website publishers with regard to their smart header bidding solutions and their connected supply-side platforms. We outline the potential of header bidding for digital service innovations around content monetization and discuss three concerns - securing the ad quality by tackling fraud, reducing latency, and coping with Google's dominant market position. Finally, we summarize our findings, point to some study limitations, and outline several paths for future research.

\section{RESEARCH BACKGROUND}

\section{Setting the Stage}

Selling and buying online ads via ad exchanges (central marketplaces) emerged when matching publishers and advertisers via direct transactions became too complex. Early on, publishers only allocated advertising inventory that did not sell directly towards real-time auction bidding (Sayedi, 2018).

A real-time auction is initiated once a user navigates to a website. User and session characteristics (e.g., demographics, location, device type, and browsing history) are transmitted to an ad exchange, which forwards them to 'their' connected advertisers. Advertisers interested in a user profile can bid for the slot (Lambrecht, 2013). The advertiser with the highest bid for that ad placement wins and gets its ad rolled out to the user. The number of players on both sides of the exchange and the architecture of the exchange determine the latency of this automated process (Gonzalvez and Mochon, 2016). Real-time advertising auctions have gained ground in the efforts to cope with increasing latency and complexity (Arkhipov et al., 2016).

Waterfall auctions are still the standard mechanism for selling ads online. With waterfall auctions, publishers connect to several advertiser networks on their own server. They arrange those networks and exchanges in a fixed order based on past performances regarding fill rates, latency, and CPM prices. Advertisers further down the chain who might be interested in an impression cannot bid if a previous bid clears the price-floor. Waterfall auctions do not exploit the ad inventory based on price. Every time an impression moves down a line, its price is reduced - thereby decreasing the ad value for the publisher and not necessarily reflecting the buyer's true willingness-to-pay. Passing down an impression causes a delay on the user's website (Zawadzinski, 2018). More advertisers participating in the waterfall auction increase latency and worsen the situation (Balseiro et al., 2014).

About $25 \%$ of the top 1,000 publishing websites in the US rely on waterfall auctions (Serverbid, 2018). A large proportion of waterfall auctions take place on a central exchange platform like Google's DoubleClick for Publishers, which prefers bids from its own ad exchange (Choi, 2018; Zawadzinski, 2018).

\section{Foundations of Header Bidding Research}

Header bidding offers a rather new service of real-time bidding auctions in publishers' efforts to address complexity and latency issues and thereby tackle Google's sector dominance. It is grounded in five research areas.

Service digitization. Research on service digitization takes a broad view on the industry or ecosystem level and has investigated how digitization-enabled phenomena such as the Internet of Things, cloud(-sourcing), Big Data, and Big Data Analytics impact services. On the micro-level, service digitization can fundamentally change or supersede human labor around data and nonroutine processes (Rifkin, 2014) to the extent that it is still required in online advertising markets. On the macro-level, digitization disrupts service ecosystems and value chains. Services and business processes become commoditized (Markus and Loebbecke, 2013). Lower transaction costs threaten service intermediaries that formerly internalized those costs to provide aggregation, trust, facilitation, and matching services for market participants (Bailey and Bakos, 1997).

Smart contracts. The mechanisms for bargaining and control in header bidding resemble smart contracts as discussed in the context of smart and autonomous technologies. A smart contract is a computerized transaction protocol that executes the terms of a contract (Avital, 2018; Murray et al., 2019); it minimizes the need for trusted intermediaries between transacting parties (Christidis and Devetsikiotis, 2016). Smart contracts are mostly relevant in the context of blockchain technology (Beck et al., 2018; Murray et al., 2019). They also gain importance in auctions (Hahn et al., 2017) and the publishing of ads (Bartoletti and Pompianu, 2017).

Real-time bidding. Real-time bidding constitutes another foundation for the study of header bidding. Often, it is combined with machine learning to compute how relevant an impression is for an advertiser based on diverse user and session features (Lacerda et al., 2006). Research on real-time bidding investigates auctions in ad networks as the dominant form for allocating impressions. It analyzes how advertisers can optimize their bids (Ren et al., 2018), how publishers can allocate their advertising space and maximize revenue (Balseiro et al., 2014), and how equilibrium advertisers' and publishers' strategies together drive equilibrium profits (Sayedi, 2018). Finally, it lays out how and with what granularity demand-side platforms should segment real-time bidding advertising markets (Qin et al., 2017).

Display advertising auctions. Early forms of programmatic display advertising comprise selling impressions in auctions and reservation contracts (Sayedi, 2018). While header bidding only relies on auctions, basic mechanisms for the allocation of display 
advertisements - that ad customization can improve the effectiveness of display advertising (Bright and Daugherty, 2012) - remain the same. Publishers can benefit from dynamic auction designs for display advertising auctions. The auction design needs to account for the trade-off between extracting the revenue from the spot markets and paying the penalty for not serving the desired advertising contacts - neither quantitatively nor qualitatively (Chen, 2017). It provides mechanisms to overcome the dilemma that advertisers often prefer bids on a cost-per-click (CPC) basis, while publishers may prefer payment on a CPM basis (Cavallo et al., 2015).

Advertisement targeting. Header bidding increases mutual access between advertisers and publishers; thereby it fosters the diversity of possible contacts and may ultimately lead to better ad targeting. From the advertisers' perspective, better targeting renders marginal consumers more profitable. Without targeting, advertisers would focus on the average consumer (Anderson and Coate, 2005). Online advertising allows for location-based targeting and data-driven user profiling (Aslam and Karjaluoto, 2017). With regard to the impact of targeting on publishers' revenue, past research provides ambiguous results. Some studies find that targeting increases competition and thereby reduces publisher revenues (Kox et al., 2017), while other studies show that targeting can increase revenues, at least in case of a sufficiently large number of serious bidders for an impression (Hummel and McAfee, 2016). Targeting can benefit publishers' profit. When a sufficiently large fraction of impressions is sold in reservation contracts, targeting positively contributes to revenue from impressions sold via real-time bidding (Sayedi, 2018). Targeting also positively contributes to advertisers' revenues as it softens competition (Chen et al., 2001; lyer et al., 2005). However, targeting can also lead to lower prices for impressions and contacts (Kox et al., 2017). Further, it raises privacy concerns among internet users, who increasingly refuse to disclose their personal information (Goldfarb and Tucker, 2012). Offering users a choice between a lowprivacy, high-targeting regime and a high-privacy, low-targeting regime maximizes consumer surplus (Kox et al., 2017).

\section{HEADER BIDDING: PROCESS, WRAPPERS, AND PLATFORMS}

Both, publishers and advertisers benefit from header bidding auctions as they reveal the true value of a single impression. Advertisers gain access to additional advertising space; they can better target their bids based on user and session characteristics. Thus, they can allocate the budget more cost-efficiently. Without a pre-determined order, all bids for impressions are considered concurrently. The resulting competition for a single impression drives impression prices, which in turn benefits publishers.

\section{Header Bidding Process}

When a user visits a website, a JavaScript code in the publisher's website initiates the header bidding. Backed by several technological innovations (Yuan et al., 2014), the bid request goes to multiple bidders at once. Publishers concurrently request multiple bids for the available ad space and compare the bids to the price previously reserved by direct contracts. The ad with the overall highest price wins. Figure 1 depicts the process of a header bidding auction.

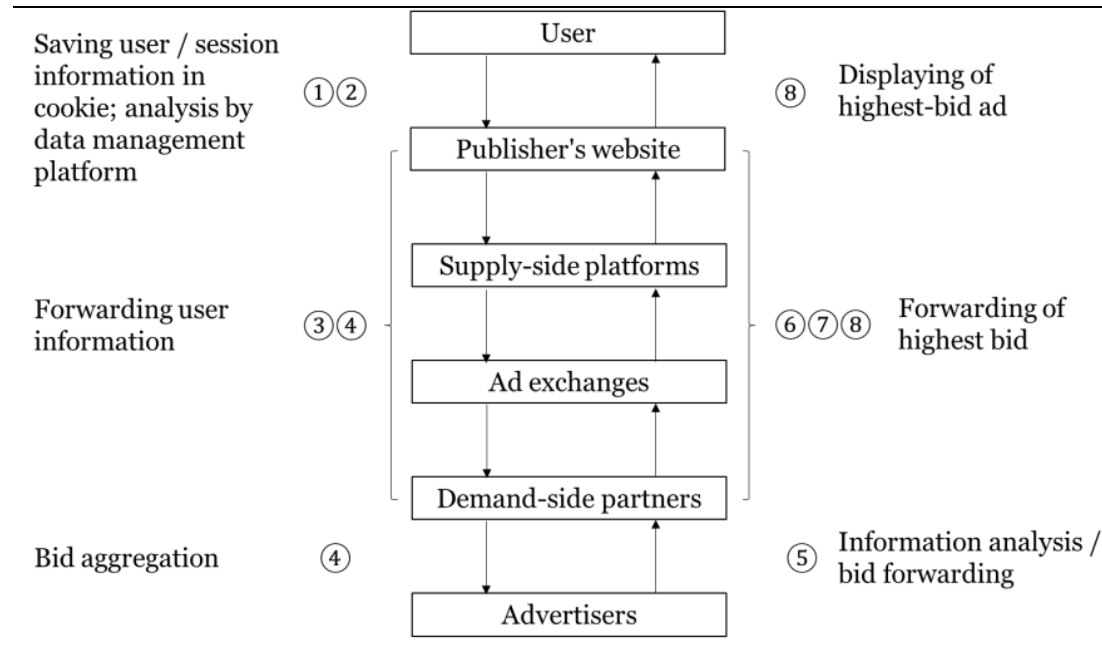

Upon a user's visit, the publisher's website stores user and session / interaction characteristics (e.g., user demographics, location, device type) in a cookie.

A data management platform analyzes the cookie data to enable advertisers to target users.

A script on the publisher's website automatically forwards the information about a user session to multiple supply-side platforms.

Each supply-side platform sends the information to several ad exchanges; each ad exchange forwards it to their connected demand-side partners. [Demand-side partners aggregate advertisers' bids as supply-side platforms collect them for publishers (Gonzalvez \& Mochon, 2016).]

A demand-side partner analyzes the information and collects the bids from connected advertisers, who are interested in targeting the user session.

The demand-side partner sends the highest advertiser bid to the ad exchanges.

The ad exchange compares the bids from all demand-side partners, determines the highest bid, and informs the supply-side platform.

The supply-side platform sends the ad to the publisher and the advertiser with the highest bid for the combination of ad space and user / session gets displayed on the user's screen.

Figure 1. Header Bidding Process (Source: Own Analysis) 
Users see a blank publisher's website until the auction closes (Balseiro and Candogan, 2017). The duration of the header bidding auction depends on the number of players involved. The ad appears as soon as the page fully loads (Adikari and Dutta, 2015).

\section{Header Bidding Wrappers}

On the website, publishers implement header bidding in the form of a header bidding wrapper - a container that enables the concurrent adding and managing of multiple auction partners making sure that no bidder can block others from bidding. Wrappers collect demand-side partners' bids and forward them to the ad exchange.

Once implemented, header bidding wrappers take pre-set parameters - such as price-floors for individual partners or a universal timeout threshold for each auction - and deploy them across all supported supply-side platforms. Furthermore, they can add or remove bidders to the auction to improve yield continually, revenue, and latency (Kneen, 2016).

Publishers can choose between open third-party / proprietary header bidding wrappers.

- Open header bidding wrappers are typically open-source and, apart from implementation costs, free of charge for any publisher. They typically allow for connecting to an extensive selection of supply-side platforms serving channels with different advertising formats (Chowla, 2018). They grant publishers full control over the bidding process and allow them to monitor and change the process (Goel, 2017). However, when implementing open header bidding wrappers, publishers must rely on their own development team, the open-source community, or external consulting firms (Kneen, 2016).

- Third-party / proprietary header bidding wrappers offer access to fewer supply-side platforms. In return, they certify each of their supply-side platforms and ad exchanges (Chinn, 2017). As they operate for profit (Burkard et al., 2012; lansiti and Levien, 2004), they typically offer value-added services such as usability and enterprise support, which come at a price to publishers.

Furthermore, publishers can select client-side, server-side, and hybrid header bidding wrappers.

- A client-side (or browser-side) wrapper loads in the user's web browser. The browser sends out multiple bid requests concurrently and the highest bid wins the auction. Every added partner increases the load time of the ad (Binns, 2018).

- A server-side wrapper activates the bid request to a single server once a user visits a website. The server forwards the bid request to all partners (Kneen, 2017) and takes the entire workload, regardless how many partners the publisher adds. However, the server does not have direct access to the user cookie data, which reduces the accuracy of targeting a user's interests (Davies, 2017).

- A hybrid wrapper combines the approaches of client-side and server-side wrappers.

\section{Header Bidding Supply-Side Platforms}

Supply-side platforms connect publishers to ad exchanges, which connect them to various demand-side ad platforms. They aggregate bids for advertising space and across different formats such as web banners, frame ads, floating ads, and sponsored stories.

Supply-side platforms analyze the available ad impressions against their advertisers' target group (Gonzalvez and Mochon, 2016). They select the highest bid from their advertisers and forward it in real time to the header bidding wrapper for inclusion in the auction (Mukherjee et al., 2017). By increasing the number of potential buyers for ad spaces, supply-side platforms enable publishers to realize higher prices (Marshall, 2014).

For publishers, choosing the right supply-side platform is a crucial decision. According to Graham (2015), the main selection criteria for selecting header bidding supply-side platforms are (1) the deployed technology, (2) the way they tackle ad fraud, (3) the supported ad formats, and (4) the technical support they provide (Graham, 2015).

\section{RESEARCH APPROACH}

First, we determined the top 20 German website publishers via industry rankings published online. We opted for using the IVW (2018) listing from Germany and selected the number of page visits as the criterion for publisher size. Second, we identified the five globally, most commonly used header bidding wrappers. Together, they cover all header bidding activities conducted by the top 20 German website publishers (Datanyze, 2018). Third, we pinpointed the supply-side platforms connected to the top 20 German website publishers. To this end, we listed all 162 supply-side platforms available to publishers worldwide (Prebid, 2018). We then applied the freely downloadable analytics tool 'Headerbid Expert' (see Figure 2) to each of the five header bidding wrappers. Subsequently, we cross-referenced the supply-side platforms with the top 20 website publishers.

Having determined the website publishers, the header bidding wrappers, and the supply-side platforms for our research, two of the authors separately conducted an in-depth analysis of their differences, as well as the benefits and drawbacks for publishers. In a face-to-face session, we synthesized the results. In two cases of disagreement, we resolved ambiguity via discussion and additional online research. 
Headerbid Expert found 2 bidders

@www.spiegel.de

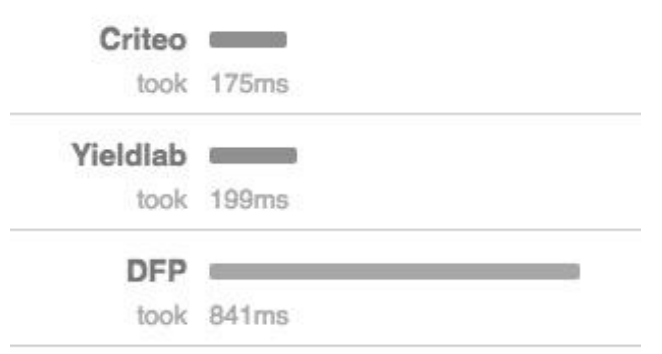

Page Analysis

Powered By 1 AppNexus

Figure 2. Example Analysis with 'Headerbid Expert' (Source: Own Analysis)

In the same team of three, we analyzed the five identified header bidding wrappers from a publisher's perspective. We then investigated the identified supply-side platforms used by the top 20 publishers in Germany. As prior research does not provide a framework for comparing supply-side platforms, we relied on the four criteria provided by Graham (2015). We added the protection of the ad quality as fifth criterion, which refers to the degree that an ad meets the end-user expectations. Thereby, we ended up with the following five criteria.

- Technology deployed (offering different degrees of access and control),

- Ad protection tackling ad fraud occurs either when an impression is sent to a bot, thereby reducing the value of an impression or when malicious code, carrying malware and viruses, is directed to a user (Edelman, 2014; Fulgoni, 2016),

- Ad formats supported (potential advertising formats include banner advertising on a desktop or mobile website to native advertising, video advertising, or ad applications for mobile phones or smart TVs),

- Technical support (for handling any problems with integrating or maintaining their platform; it differs regarding scope, possible extra fees, and language),

- Ad quality (meeting users' expectations).

\section{RESULTS}

The industry ranking provided by IVW (2018) and presented on statista.de provides us with the top 20 German website publishers (see Table 1) as a starting point for assessing commonly employed header bidding wrappers and supply-side platforms.

Table 1. Top 20 German Website Publishers (Source: IVW, 2018)

\begin{tabular}{|c|c|c|}
\hline Publisher & Category & URL \\
\hline eBay Kleinanzeigen & Private Auctions & ebay-kleinanzeigen.de \\
\hline Web.de & ISP & web.de \\
\hline T-Online & Telco Provider & t-online.de \\
\hline GMX & ISP & gmx.net \\
\hline Bild & Newspaper & bild.de \\
\hline Spiegel Online & Magazine & spiegel.de \\
\hline Focus Online & Magazine & focus.de \\
\hline Wetter.com & Weather App & wetter.com \\
\hline WetterOnline & Weather App & wetteronline.de \\
\hline Welt & Newspaper & welt.de \\
\hline $\mathrm{n}-\mathrm{tv}$ & TV News Channel & n-tv.de \\
\hline Zeit & Newspaper & zeit.de \\
\hline Süddeutsche Zeit. & Newspaper & sueddeutsche.de \\
\hline Stern & Magazine & stern.de \\
\hline Frankfurter Allg. & Newspaper & faz.net \\
\hline Express & Newspaper & express.de \\
\hline RP Online & Newspaper & rp-online.de \\
\hline Handelsblatt & Newspaper & handelsblatt.com \\
\hline tz.de & Newspaper & tz.de \\
\hline Huffpost & Newspaper & huffpost.com \\
\hline
\end{tabular}




\section{Header Bidding Wrappers}

For the above listed website publishers, we identified five header bidding wrappers which together account for all header bidding activities on Germany's top 20 publishing websites. The five wrappers are Prebid, OpenX Bidder, AppNexus, Amazon's Transparent Ad Marketplace (TAM), and Sovrn's Header Suite. Other ad tech companies offer similar services. Their offerings mainly differ in the available supply-side platforms - many of which are used only by publishers in the United States.

We differentiate the five header bidding wrappers according to two criteria, (1) the architecture / positioning and (2) the distribution model (see Table 2). Both criteria directly impact the publishers' business model: The five wrappers together cover all wrapper variations available to publishers.

Table 2. Header Bidding Wrappers Used by the Top 20 German Website Publishers (Source: Own Analysis)

\begin{tabular}{cccccc}
\hline & Prebid & OpenX Bidder & AppNexus & Amazon's TAM & Sovrn's Header Complete \\
\hline Architecture / Positioning & Hybrid & Client-Side & Hybrid & Hybrid & Client-Side \\
\hline Distribution Model & Open & 3rd Party / Proprietary & 3rd Party / Proprietary & 3rd Party / Proprietary & 3rd Party / Proprietary \\
\hline
\end{tabular}

Concerning the architecture / positioning, client-side and server-side wrappers each account for about $40 \%$ and hybrid wrappers for the remaining $20 \%$ among German publishers (Serverbid, 2018). Globally, the latter have experienced the most significant growth in 2018 (Serverbid, 2018).

A more detailed analysis shows relevant differences among header bidding wrappers influencing ng a publisher's business model.

- Prebid, available as client-side and as server-side wrapper, is the most popular open-source header bidding wrapper in Germany with more than 13,000 installations. Created by AppNexus engineers (Pichardo, 2017) and based on Prebid Core, it is designed to enable the simplest form of header bidding. Prebid Core handles the entire header bidding process from initiating the bid request, collecting incoming bids, timing out, and comparing the bids. It sends the highest bid to the publisher's advertising server and logs all data for later reporting. Prebid Core offers asynchronous bidding, unified data collection, and the ability to set a central timeout. Free to implement, Prebid provides publishers with a high degree of transparency; they can see participating bidders, targeting information, incoming bids, and the ads displayed on the user's web browser.

- OpenX Bidder, among the first to provide a header bidding solution to publishers (Sluis, 2015), is a client-based wrapper which achieves an average of 62 billion bids daily with a 95\% participation rate (OpenX, 2018). More than 76,600 websites in Germany use OpenX services (SimilarTech, 2018), making it the top-ranked header bidding technology in Germany with $66 \%$ market share (Datanyze, 2018). OpenX integrates a supply-side platform and a header bidding wrapper. Its own ad exchange handles every bid request. Publishers can neither choose the number of supply-side platforms nor decide which potential buyers participate in the header bidding auction.

- AppNexus, available as client-side and as server-side wrapper, uses the open-source header bidding wrapper Prebid. It offers a holistic approach for various direct and exchange-based, stationary and mobile channels. It provides display and video of ad content (Timm, 2017). With AppNexus, publishers review all auction dynamics: they can take advantage of the analytics engine Yieldex for setting the price for their ad spaces using predictive forecasting (Kosoff, 2015).

- Amazon's Transparent Ad Marketplace (TAM), a server-side technology, was introduced in Germany in January 2018. With about $20 \%$ market share, they became number two in the market after less than a year. Publishers can activate multiple supply-side platforms including Amazon itself; every bid is treated equally (Amazon, 2018). Publishers enjoy full transparency as they can see metrics such as CPM, rates of bidding, rates of winning, and the latency of every participating supply-side platform. Bidders pay \$0.01 CPM to Amazon and publishers receive $100 \%$ of the winning bid.

- Sovrn's Header Complete is third in the German market with about 7\% market share (Datanyze, 2018). Built upon Prebid, it offers a single implementation, full service, and a unified report on connected supply-side platforms. Further, it offers access to about twenty integrated supply-side platforms and promises a maximum response time of 240ms (Sovrn, 2018). Publishers receive a percentage from displaying an ad, which depends on a pre-determined revenue share model (Graham, 2015).

\section{Header Bidding Supply-Side Platforms}

The top 20 German website publishers integrate ten different supply-side platforms. On average, the publishers work with about two platforms (see Table 3), whereas the average number of supply-side platforms among the top 1,000 websites in the US is almost six (Serverbid, 2018). 
Table 3. Supply-Side Platforms Used by the Top 20 German Website Publishers (Source: Own Analysis in May 2018)

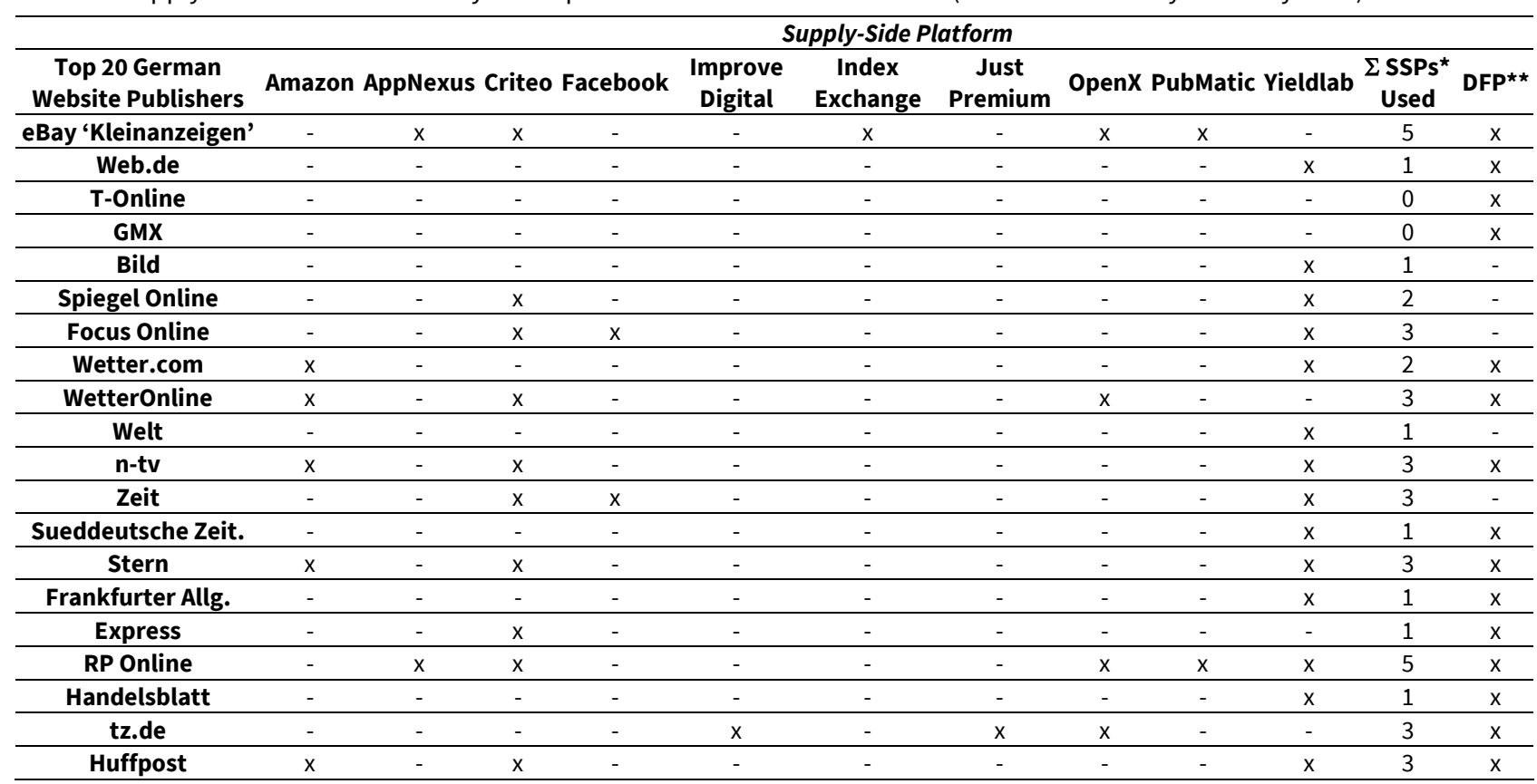

* Supply-Side Platform ** Google's DoubleClick for Publishers

The ten supply-side platforms cover a wide range of advertising formats ranging from simple display advertising across desktop and mobile browsers to more sophisticated variants such as native, video, or rich media advertising. Each platform proposes its own method to 'guarantee' a brand-safe environment for publishers. However, the industry lacks a standard regarding the quality and security of advertising.

Fourteen of 20 analyzed German publishers choose Yieldlab as a supply-side platform; it is the only one based in Germany. Of those, five publishers select Yieldlab as their only supply-side platform next to Google's DoubleClick for Publishers. Table 4 summarizes our analysis of the ten supply-side platforms.

Table 4. Supply-Side Platforms Used by the Top 20 Publisher Websites in Germany (Source: Own Analysis)

\begin{tabular}{|c|c|c|c|c|c|}
\hline \multirow[b]{2}{*}{$\begin{array}{l}\text { Supply-Side } \\
\text { Platform (SSP) }\end{array}$} & \multicolumn{5}{|c|}{ Criterion } \\
\hline & Technology Deployed & $\begin{array}{l}\text { Ad Protection (Tackling } \\
\text { Ad fraud) }\end{array}$ & $\begin{array}{l}\text { Ad Formats } \\
\text { Supported }\end{array}$ & $\begin{array}{l}\text { Technical } \\
\text { Support }\end{array}$ & Ad Quality \\
\hline Amazon & Unified Ad Marketplace & Automated review & $\begin{array}{c}\text { Display on desktop, } \\
\text { mobile }\end{array}$ & Online support & $\begin{array}{l}\text { Unique Amazon ads and } \\
\text { third-party auto-reviewed }\end{array}$ \\
\hline AppNexus & Publisher SSP & $\begin{array}{l}\text { Automated in-house } \\
\text { review }\end{array}$ & $\begin{array}{l}\text { Display, video, } \\
\text { native on desktop } \\
\text { and mobile }\end{array}$ & $\begin{array}{c}\text { Dedicated account } \\
\text { manager }\end{array}$ & $\begin{array}{l}\text { Manual review through } \\
\text { mobile app for publishers }\end{array}$ \\
\hline Criteo & $\begin{array}{c}\text { Direct Bidder and } \\
\text { Publisher Marketplace }\end{array}$ & $\begin{array}{l}\text { Manual and Integral Ad } \\
\text { Science }\end{array}$ & Display and native & $\begin{array}{c}\text { Online support and } \\
\text { dedicated account } \\
\text { manager }\end{array}$ & Acceptable ads program \\
\hline Facebook & Audience Network & $\begin{array}{c}\text { Automated and manual } \\
\text { review }\end{array}$ & $\begin{array}{l}\text { Display and native } \\
\text { on mobile }\end{array}$ & $\begin{array}{l}\text { Online support } \\
\text { (development \& } \\
\text { management) }\end{array}$ & $\begin{array}{l}\text { Filter for individual } \\
\text { categories or formats }\end{array}$ \\
\hline Improve Digital & $\begin{array}{c}\text { Header Bidding, server- } \\
\text { side and Full Holistic }\end{array}$ & Integral Ad Sciences & $\begin{array}{c}\text { Display, video, } \\
\text { native, rich media }\end{array}$ & $\begin{array}{c}\text { Dedicated account } \\
\text { manager }\end{array}$ & $\mathrm{n} / \mathrm{a}$ \\
\hline Index Exchange & $\begin{array}{c}\text { Client-side and server- } \\
\text { side SSP }\end{array}$ & $\begin{array}{c}\text { Trustworthy Accountability } \\
\text { Group (TAG) }\end{array}$ & $\mathrm{n} / \mathrm{a}$ & $\begin{array}{c}\text { Dedicated account } \\
\text { manager, German office }\end{array}$ & Manual review of every ad \\
\hline Just-Premium & Out-of-Page-Room & $\mathrm{n} / \mathrm{a}$ & $\begin{array}{c}\text { Skins, footer, } \\
\text { header, scroller }\end{array}$ & $\begin{array}{c}\text { Dedicated account } \\
\text { manager, German office }\end{array}$ & Manual review \\
\hline OpenX & $\begin{array}{l}\text { Client-side and server- } \\
\text { side SSP with demand } \\
\text { fusion }\end{array}$ & $\begin{array}{c}\text { Trustworthy Accountability } \\
\text { Group (TAG), Integral Ad } \\
\text { Sciences }\end{array}$ & $\begin{array}{l}\text { Display, video, } \\
\text { native on desktop } \\
\text { and mobile }\end{array}$ & $\begin{array}{l}\text { Online documentation } \\
\text { and premium services }\end{array}$ & $\begin{array}{l}\text { Proprietary system with } \\
\text { manual review }\end{array}$ \\
\hline PubMatic & $\begin{array}{l}\text { Client-side SSP and own } \\
\text { header bidding wrapper }\end{array}$ & $\begin{array}{l}\text { Fraud-free rewards } \\
\text { program }\end{array}$ & $\begin{array}{l}\text { Display, video, } \\
\text { native on desktop } \\
\text { and mobile }\end{array}$ & $\begin{array}{c}\text { Online support } \\
\text { community (resources } \\
\text { and forum) }\end{array}$ & $\begin{array}{l}\text { In-house algorithm and } \\
\text { external partners }\end{array}$ \\
\hline Yieldlab & $\begin{array}{l}\text { Client-side SSP, } \\
\text { Yieldprobe }\end{array}$ & In-house operations team & $\begin{array}{l}\text { Display, video, } \\
\text { native on desktop } \\
\text { and mobile }\end{array}$ & $\begin{array}{l}\text { Self-service, assistance, } \\
\text { managed service }\end{array}$ & $\begin{array}{l}\text { White-listing and black- } \\
\text { listing for publishers }\end{array}$ \\
\hline
\end{tabular}




\section{DISCUSSION}

With our research, we show the business potential of header bidding as service innovation in the digital era. As deploying new technology and growing service and business model options are deeply intertwined (Al-Debei and Avison, 2010; Knop et al., 2017; Loebbecke and Picot, 2015; Zott and Amit, 2008), publishers must choose a header bidding technology which matches their resources and technical know-how (McLaren et al., 2011). They must find solutions - likely technical in nature - to two concerns: securing the ad quality by tackling fraud and reducing latency - both major arguments for publishers' own header bidding solutions (Levine, 2017). Further, they must find a way to cope with Google as market dominator.

- Securing the quality of displayed ads remains a major concern among publishers; it supports the business case of header bidding wrappers and other intermediates but reduces the market efficiency for publishers. The traditional CPM 'currency' barely delivers the needed information (Bandyopadhyay et al., 2009). A comparable 'currency' for online ads is missing; the lack of an agreed-upon standard for defining and measuring ad quality worsens the problem.

A solution based on blockchain technology (Beck et al., 2018; Avital, 2018) substituting for the intermediary or trusted third-party comes to mind (Anjum et al., 2017; Maglio et al., 2015). Transactions on ad exchanges are mostly complete contracts, fostering the idea of introducing a blockchain-based solution mediating in the online ad market (Beverungen et al., 2019) - even if any such blockchain-based solution appears to be difficult due to latency concerns. Further, Artificial Intelligence (AI) based applications may help to tackle ad fraud (Makridakis, 2017; McAfee and Brynjolfsson, 2017).

It does not help much that other sectors also struggle with securing data quality, here 'ad quality'. For instance, the open science community promotes data quality requirements. To be eligible for public funding, increasingly data must be FAIR that is Findable, Accessible, Interoperable, and Re-usable (Wilkinson et al., 2016). The open science community widely approves those data FAIRness principles - yet so far without agreement on how to measure and operationalize them.

- Latency concerns quickly resurface with the growing popularity of header bidding. Adding more supply-side platforms to a header bidding wrapper increases latency and thus risks the monetization potential. No server-side header bidding fully cures for latency. Latency concerns also limit the potential of any blockchain-based smart contract solution to assure ad quality and tackle ad fraud. It remains to be seen whether the industry will find a business model which is less demanding with respect to latency or can count on even faster CPUs and IT / telecommunication networks.

- Finally, publishers need to account for Google's dominance in the ad market with its decreasing prices for ad impressions. Google, with its acquisition of DoubleClick in 2008, first integrated services for ad buying and selling at low to no cost; it tightly coupled its ad server to its demand platform. In early 2017, Google introduced its Exchange Bidding Dynamic Allocation, which integrates auction and ad serving and thereby decreases the latency problem by eliminating a former two-step process. Google's platform could soon impose quasi-standard commoditized processes (Davenport, 2005) and act as a self-appointed orchestrator of supply-side platforms, ad exchanges, and advertiser networks (Markus and Loebbecke, 2013).

Over the last fifteen years, publishers' investments in developing and promoting header bidding solutions have paid off. However, they will have to stay at the forefront of technology and work with supply-side platforms to further develop header bidding or come up with complementing smart service innovations that let publishers flourish side-by-side with a market dominator.

\section{SUMMARY, LIMITATIONS, AND FUTURE RESEARCH}

The goal of this paper was to analyze the requirements for successful header bidding and to present the opportunities and challenges arising from implementing header bidding as publishers' main business model for display advertising. To this end, we collected data on the top 20 German website publishers, header bidding wrappers, and the supply-side platforms employed by the publishers. We analyzed the header bidding wrappers and the supply-side platforms to draw conclusions on publishers' required business model transformation for selling online ads. We have offered some guidance on how to take advantage of the 'header bidding' wave of monetizing publishers' online content and raised some concerns on how sustainable the current header bidding success will likely be in light of Google's Exchange Bidding.

Approaching the header bidding phenomenon from actual market implementations, our research faces two main limitations. Firstly, we limit our investigation to the five header bidding wrappers deployed by the top 20 German website publishers. Other wrappers may offer technological features, which translate into additional business model opportunities. Secondly, we assessed our initial set of globally available 162 supply-side platforms as being too large for a comprehensive analysis. Reducing the overall set to the ten supply-side platforms, which are used by the top 20 German publishers, may carry some geographical bias; it cannot prescribe the 'best' set of supply-side platforms available to German publishers. From these limitations, we suggest several paths for complementary work.

Future research might want to propose a research framework based on the service innovation literature to substantiate this research further on a theoretical level. Future research might also want to investigate in more detail the impact of combining client-side and server-side header bidding wrappers with regard to publishers' required implementation, maintenance, and optimization efforts. It could also focus on technical solutions for integrating more supply-side platforms and investigate the 'yield break-even' between latency and price increases due to competition or between latency and trust provision in the context of ad fraud. A case study - ideally comparing various header bidding scenarios in one publisher's business ecosystem - might help discover additional challenges and opportunities not discovered by this research. Another promising avenue for future research 
is the investigation of privacy and security concerns for analyzing the identified solutions from a publisher's perspective. Finally, reaching out to recent technology deployments, we see potential for two major research streams: Firstly, the integration of Albased service offerings on the wrapper side deserves critical business model analyses; it needs to keep in mind the dependency on available (user) data for machine learning scenarios. Secondly, researchers might be keen on designing and deploying blockchain-based solutions for online ad exchanges - enjoying the complete contracts when having to balance industry concerns regarding latency and trust provision.

\section{REFERENCES}

Adikari, S. and Dutta, K. (2015). Real-Time Bidding in Online Digital Advertisement. In B. Donnellan, M. Helfert, J. Kenneally, D. VanderMeer, M. Rothenberger and R. Winter (eds.), New Horizons in Design Science: Broadening the Research Agenda (pp. 1938). Cham, Switzerland: Springer International Publishing, DESRIST 2015, Lecture Notes in Computer Science, vol. 9073. https://doi.org/10.1007/978-3-319-18714-3_2

Al-Debei, M. and Avison, D. (2010). Developing a Unified Framework of the Business Model Concept. European Journal of Information Systems, 19(3), 359-376. https://doi.org/10.1057/ejis.2010.21

Amazon (2018). Transparent Ad Marketplace. Available at: https://aps.amazon.com/aps/transparent-ad-marketplace (Accessed April 11, 2019).

Anderson, S. and Coate, S. (2005). Market Provision of Broadcasting: A Welfare Analysis. Review of Economic Studies, 72(4), $947-972$. https://doi.org/10.1111/0034-6527.00357

Anjum, A., Sporny, M. and Sill, A. (2017). Blockchain Standards for Compliance and Trust. IEEE Cloud Computing, 4(4), 84-90. https://doi.org/10.1109/MCC.2017.3791019

Arkhipov, D., Turner, J., Dillencourt, M., Torres, P. and Regan, A. (2016). Yield Optimization with Binding Latency Constraints, in 3rd International Conference on Soft Computing and Machine Intelligence (ISCMI), Dubai, UAE, 5-12. https://doi.org/10.1109/ISCMI.2016.51

Aslam, B. and Karjaluoto, H. (2017). Digital Advertising around Paid Spaces, E-Advertising Industry's Revenue Engine: A Review and Research Agenda. Telematics and Informatics, 34(8), 1650-1662. https://doi.org/10.1016/j.tele.2017.07.011

Avital, M. (2018). Peer Review: Toward a Blockchain-Enabled Market-Based Ecosystem. Communications of the Association for Information Systems, (42), 646-653. https://doi.org/10.17705/1CAIS.04228

Bailey, J. and Bakos, Y. (1997). An Exploratory Study of the Emerging Role of Electronic Intermediaries. International Journal of Electronic Commerce, 1(3), 7-20. https://doi.org/10.1080/10864415.1997.11518287

Balseiro, S. and Candogan, O. (2017). Optimal Contracts for Intermediaries in Online Advertising. Operations Research, 65(4), 878896. https://doi.org/10.1287/opre.2017.1618

Balseiro, S., Feldman, J., Mirrokni, V. and Muthukrishnan, S. (2014). Yield Optimization of Display Advertising with Ad Exchange. Management Science, 60(12), 2886-2907. https://doi.org/10.1287/mnsc.2014.2017

Bandyopadhyay, S., Wolfe, J. and Kini, R. (2009). A Critical Review of Online Affiliate Models. Journal of Academy of Business and Economics, 9(4), 141-147.

Bartoletti, M. and Pompianu, L. (2017). An Empirical Analysis of Smart Contracts: Platforms, Applications, and Design Patterns. In 21st International Conference on Financial Cryptography and Data Security, Cham, Switzerland: Springer International Publishing Switzerland, 494-509. https://doi.org/10.1007/978-3-319-70278-0_31

Beck, R., Mueller-Bloch, C. and King, J. (2018). Governance in the Blockchain Economy: A Framework and Research Agenda. Journal of the Association of Information Systems, 19(10), 1020-1034. https://doi.org/10.17705/1jais.00518

Beverungen, D., Mueller, O., Matzner, M., Mendling, J. and vom Brocke, J. (2019). Conceptualizing Smart Service Systems. Electronic Markets, 29(1), 7-18. https://doi.org/10.1007/s12525-017-0270-5

Binns, A. (2018). How Moving to Server-Side Header Bidding Reduces Latency. Available at: www.pubmatic.com/blog/server-sideheader-bidding-reduces-latency (Accessed March 02, 2019).

Bright, L. and Daugherty, T. (2012). Does Customization Impact Advertising Effectiveness? An Exploratory Study of Consumer Perceptions of Advertising in Customized Online Environments. Journal of Marketing Communications, 18(1), 19-37. https://doi.org/10.1080/13527266.2011.620767

Burkard C, Widjaja T. and Buxmann, P. (2012). Software Ecosystems. Business Information Systems Engineering, 4(1), $41-44$. https://doi.org/10.1007/s12599-011-0199-8

Cavallo, R., McAfee, R. and Vassilvitskii, S. (2015). Display Advertising Auctions with Arbitrage. ACM Transactions on Economics and Computation, 3(3), 1-21. https://doi.org/10.1145/2668033

Chen, Y. (2017). Optimal Dynamic Auctions for Display Advertising. Operations Research, 65(4), 897-913. https://doi.org/10.1287/opre.2017.1592

Chen, Y., Narasimhan, C. and Zhang, Z. (2001). Individual Marketing with Imperfect Targetability. Marketing Science, 20(1), $23-41$. https://doi.org/10.1287/mksc.20.1.23.10201 
Chinn, C. (2017). 5 Elements Every Wrapper Solution Should Provide. Available at: www.pubmatic.com/blog/elements-everywrapper-solution-provide (Accessed January 07, 2019).

Choi, J. (2018). The Google Monopoly That No One Is Talking About. Available at: www.digitalcontentnext.org/blog/2018/11/02/the-google-monopoly-that-no-one-is-talking-about (Accessed April 11, 2019).

Chowla, M. (2018). Why Open Source Technology? Why Now?. Available at: www.pubmatic.com/blog/open-source-now (Accessed February 02, 2019).

Christidis, K. and Devetsikiotis, M. (2016). Blockchains and Smart Contracts for the Internet of Things. IEEE Access, (4), $2292-2303$. https://doi.org/10.1109/ACCESS.2016.2566339

Cook, J. and Attari, S. (2012). Paying for What Was Free: Lessons from the New York Times Paywall. Cyberpsychology, Behavior, and Social Networking, 15(12), 1-6. https://doi.org/10.1089/cyber.2012.0251

Datanyze (2018). OpenX Bidder Market Share in Germany and Competitor Report. Available at: www.datanyze.com/marketshare/header-bidding/Germany/openx-bidder-market-share (Accessed June 11, 2019).

Davenport, T. (2005). The Coming Commoditization of Processes. Harvard Business Review, 83(6), 100-108.

Davies, J. (2017). Lower Match Rates and Reduced Yields: Publishers' Concerns about Server-Side Header Bidding. Available at: www.digiday.com/uk/lower-match-rates-reduced-yields-publishers-concerns-server-side-header-bidding (Accessed March 06, 2019).

Edelman, B. (2014). Pitfalls and Fraud in Online Advertising Metrics: What Makes Advertisers Vulnerable to Cheaters, and How They Can Protect Themselves. Journal of Advertising Research, 54(2), 127-132. https://doi.org/10.2501/JAR-54-2-127-132

Fulgoni, G. (2016). Fraud in Digital Advertising: A Multibillion-Dollar Black Hole. Journal of Advertising Research, 56(2), $122-125$. https://doi.org/10.2501/JAR-2016-024

Goel, R. (2017). Let's Be Clear: The Transparency Debate is about Control. Available at: www.pubmatic.com/blog/lets-be-clearthe-transparency-debate-is-about-control (Accessed February 02, 2019).

Goldfarb, A. and Tucker, C. (2012). Shifts in Privacy Concerns. American Economic Review, $102(3), 349-353$. https://doi.org/10.1257/aer.102.3.349

Gonzalvez, J. and Mochon, F. (2016). Operating an Advertising Programmatic Buying Platform: A Case Study. International Journal of Interactive Multimedia and Artificial Intelligence, 3(6), 6-15. https://doi.org/10.9781/ijimai.2016.361

Graham, K. (2015). Ad Network Review: Sovrn Holdings. Available at: www.monetizemore.com/blog/ad-network-review-sovrnholdings (Accessed July 05, 2019).

Hahn, A., Singh, R., Liu, C. and Chen, S. (2017). Smart Contract-Based Campus Demonstration of Decentralized Transactive Energy Auctions. In 2017 IEEE Power and Energy Society Innovative Smart Grid Technologies Conference (ISGT), Torino, Italy, 1-5. https://doi.org/10.1109/ISGT.2017.8086092

Hummel, P. and McAfee, R. (2016). When Does Improved Targeting Increase Revenue?. ACM Transactions on Economics and Computation (TEAC), 5(1), 4. https://doi.org/10.1145/2956586

Iansiti, M. and Levien, R. (2004). Strategy as Ecology. Harvard Business Review, 82(9), 132-133.

IVW (2018). IVW Ausweisung Digitalangebote. Available at: www.de.statista.com/statistik/daten/studie/162942/umfrage/top-20internet-angebote-nach-anzahl-der-visits (Accessed May 06, 2019).

lyer, G., Soberman, D. and Villas-Boas, J. (2005). The Targeting of Advertising. Marketing Science, 24(3), 461-476. https://doi.org/10.1287/mksc.1050.0117

Kneen, B. (2016). Guide to Header Bidding Wrappers. Available at: www.adopsinsider.com/header-bidding/guide-header-biddingwrappers (Accessed February 08, 2019).

Kneen, B. (2017). Server-Side Header Bidding Explained. Available at: www.adopsinsider.com/header-bidding/server-sideheader-bidding (Accessed February 12, 2019).

Knop, S., Galipoglu, E., Lubarski, A. and Poeppelbuss, J. (2017). Quo Innovadis?: The Who, the What and the How of Research at the Intersection of ICT and Service Innovation. In 38th International Conference on Information Systems (ICIS 2017), Seoul, South Korea, 1172-1195.

Kosoff, M. (2015). New York Adtech Giant AppNexus Buys Digital Advertising and Analytics Company Yieldex for $\$ 100$ Million. Available at: www.businessinsider.com/appnexus-buys-yieldex-for-100-million-2015-3?IR=T (Accessed May 07, 2019).

Kox, H., Straathof, B. and Zwart, G. (2017). Targeted Advertising, Platform Competition, and Privacy. Journal of Economics and Management Strategy, 26(3), 557-570. https://doi.org/10.1111/jems.12200

Lacerda, A., Cristo, M., Gonçalves, M., Fan, W., Ziviani, N. and Ribeiro-Neto, B. (2006). Learning to Advertise. In 29th Annual International ACM SIGIR Conference on Research and Development in Information Retrieval, Seattle, Washington, USA, 549-556. https://doi.org/10.1145/1148170.1148265

Lambrecht, A. (2013). When Does Retargeting Work? Information Specificity in Online Advertising. Journal of Marketing Research, 50(5), 561-576. https://doi.org/10.1509/jmr.11.0503

Levine, B. (2017). Countering Header Bidding, Google Drops Its 'Last Look' Advantage. Available at: www.martechtoday.com/countering-header-bidding-google-drops-last-look-advantage-196889 (Accessed February 14, 2019). 
Loebbecke, C. and Picot, A. (2015). Reflections on Societal and Business Model Transformation Arising from Digitization and Big Data Analytics: A Research Agenda. Journal of Strategic Information Systems, 24(3), $149-157$. https://doi.org/10.1016/j.jsis.2015.08.002

Maglio, P. and Lim, C. (2016). Innovation and Big Data in Smart Service Systems. Journal of Innovation Management, 4(1), 11-21. https://doi.org/10.24840/2183-0606_004.001_0003

Maglio, P., Kwan, S. and Spohrer, J. (2015). Commentary - Toward a Research Agenda for Human-Centered service System Innovation. Service Science, 7(1), 1-10. https://doi.org/10.1287/serv.2015.0091

Makridakis, S. (2017). The forthcoming Artificial Intelligence (AI) revolution: Its impact on society and firms. Futures, 90, 46-60. https://doi.org/10.1016/j.futures.2017.03.006

Markus, M. and Loebbecke, C. (2013). Commoditized Digital Processes and Business Community Platforms: New Opportunities and Challenges for Digital Business Strategies. MIS Quarterly, 37(2), 649-653.

Marshall, J. (2014). What is a Supply-Side Platform. Available at: www.digiday.com/media/wtf-supply-side-platform (Accessed February 03, 2019).

McAfee, A. and Brynjolfsson, E. (2017). Machine, Platform, Crowd: Harnessing our Digital Future. New York, NY: W.W. Norton \& Company.

McLaren, T., Head, M., Yuan, Y. and Chan, Y. (2011). A Multilevel Model for Measuring Fit between a Firm's Competitive Strategies and Information Systems Capabilities. MIS Quarterly, 35(4), 909-929. https://doi.org/10.2307/41409966

Mukherjee, A., Sundarraj, R. and Dutta, K. (2017). Apriori Rule-Based In-App Ad Selection Online Algorithm for Improving SupplySide Platform Revenues. ACM Transactions on Management Information Systems, 8(2), 1-28. https://doi.org/10.1145/3086188

Murray, A., Kuban, S., Josefy, M. and Anderson, J. (2019). Contracting in the Smart Era: The Implications of Blockchain and Decentralized Autonomous Organizations for Contracting and Corporate Governance. Academy of Management Perspectives. https://doi.org/10.5465/amp.2018.0066

OpenX (2018). OpenX Bidder. Available at: www.docs.openx.com/Content/publishers/bidder.html (Accessed February 07, 2019).

Pichardo, K. (2017). The State of the Wrapper: Interview with AppNexus' Michael Richardson. Available at: www.pubmatic.com/blog/prebid-org-appnexus-michael-richardson-2 (Accessed March 07, 2019).

Prebid (2018). Bidders' Params. Available at: www.prebid.org/dev-docs/bidders.html (Accessed May 07, 2019).

Qin, R., Yuan, Y. and Wang, F. (2017). Exploring the Optimal Granularity for Market Segmentation in RTB Advertising via Computational Experiment Approach. Electronic Commerce Research and Applications, 24(c), 68-83. https://doi.org/10.1016/j.elerap.2017.07.001

Ren, K., Zhang, W., Chang, K., Rong, Y., Yu, Y. and Wang, J. (2018). Bidding Machine: Learning to Bid for Directly Optimizing Profits in Display Advertising. IEEE Transactions on Knowledge and Data Engineering, 30(4), 645-659. https://doi.org/10.1109/TKDE.2017.2775228

Rifkin, J. (2014). The Zero Marginal Cost Society. New York, USA: Palgrave Macmillan.

Sayedi, A. (2018). Real-Time Bidding in Online Display Advertising. Marketing Science, 37(4), 553-568. https://doi.org/10.1287/mksc.2017.1083

Serverbid (2018). Header Bidding Industry Index (HBIX). Available at: www.serverbid.com/hbix (Accessed March 01, 2019).

Shirky, C. (2008). Here Comes Everybody: The Power of Organizing without Organizations. London, United Kingdom: Penguin Books. https://doi.org/10.1038/hdy.2008.68

SimilarTech (2018). Market Share \& Web Usage Statistics OpenX. Available at: www.similartech.com/technologies/openx (Accessed April 05, 2019).

Sluis, S. (2015). The Rise of 'Header Bidding' and the End of the Publisher Waterfall. Available at: www.adexchanger.com/publishers/the-rise-of-header-bidding-and-the-end-of-the-publisher-waterfall (Accessed October 14, 2018).

Sovrn (2018). Invoke All Your Header Bidding Partners with One Integration. Available at: www.sovrn.com/product-feature/invokeheader-bidding-partners-one-integration (Accessed April 04, 2019).

Timm, F. (2017). Google Dominiert Den Deutschen Adtech-Markt. Available at: www.adzine.de/2017/04/google-dominiert-dendeutschen-adtech-markt (Accessed November 14, 2018).

Wilkinson, M., Dumontier, M., Aalbersberg, I., Appleton, G., Axton, M., Baak, A. and Blomberg, N. (2016). The FAIR Guiding Principles for Scientific Data Management and Stewardship. Scientific Data, 3(1), 1-9. https://doi.org/10.1038/sdata.2016.18

Yuan, Y., Wang, F., Li, J. and Qin, R. (2014). A Survey on Real Time Bidding Advertising. In Proceedings of 2014 IEEE International Conference on Service Operations and Logistics, and Informatics, Qingdao, China, $418-423$. https://doi.org/10.1109/SOLI.2014.6960761

Zawadzinski, M. (2018). Waterfalling, Header Bidding and New Auction Dynamics. Available at: www.clearcode.cc/blog/sequentialauctions-header-bidding-first-price-second-price-auctions (Accessed March 07, 2019).

Zott, C. and Amit, R. (2008). The Fit between Product Market Strategy and Business Model. Strategic Management Journal, 29(1), 1-26. https://doi.org/10.1002/smj.642 